\title{
Educação Permanente em Saúde Aplicada aos Técnicos no Setor de Hemoterapia de um Hemocentro Regional
}

\section{Permanente Health Education Applied to Technicians in the Hemotherapy Sector off a Regional Blood Center}

\section{Educación Permanente en Salud Aplicada a Técnicos en el Sector de Hemoterapia de un Centro Re- gional de Sangre}

\section{Milena Marques Dias ${ }^{1 *}$, Benedito Carlos Cordeiro}

Como citar esse artigo. Dias, MM; Cordeiro, BC. Educação Permanente em Saúde Aplicada aos Técnicos no Setor de Hemoterapia de um Hemocentro Regional. Revista Pró-UniverSUS. 2021 Jan./Jun.; 12 (1): 70-72.

\section{Resumo}

Nota prévia de uma pesquisa que tem como objetivo propor um programa de Educação Permanente em Saúde aos técnicos responsáveis pelos exames imunohematológicos do Hemocentro de Niterói, localizado do Hospital Universitário Antônio Pedro, a partir de uma investigação com abordagem metodológica qualitativa do tipo descritiva, com natureza exploratória. A pesquisa obteve parecer favorável do Comitê de Ética em Pesquisa da Universidade Federal Fluminense. A coleta de dados será realizada por meio de questionários semiestruturados. Os resultados serão analisados conforme categorização e análise de conteúdo proposta por Bardin. Como resultado pretende-se, através da Educação Permanente em Saúde, proporcionar aos profissionais técnicos em hemoterapia novas maneiras de pensar e solucionar os problemas surgidos no cotidiano do trabalho, com a finalidade de aumentar a qualidade do serviço prestado e a segurança do paciente atendido.

Palavras-chave: Transfusão de Sangue; Serviço de Hemoterapia; Educação Continuada.

\begin{abstract}
Preliminary note from a research that aims to propose a Permanent Health Education program to the technicians responsible for immunohematological examinations of the Niterói Hemocenter, located at the University Hospital Antonio Pedro, from a research with qualitative methodological approach of descriptive type, with nature exploratory. The research obtained a favorable opinion from the Research Ethics Committee of the Fluminense Federal University. Data collection will be performed through semi-structured questionnaires. The results will be analyzed according to categorization and content analysis proposed by Bardin. As a result, it is intended, through Permanent Health Education, to provide technical professionals in hemotherapy new ways of thinking and solving the problems that arise in daily work, with the purpose of increasing the quality of service provided and the safety of the patient being treated.

Keywords: Blood Transfusion; Hemotherapy Service; Continuing Education.
\end{abstract}

\section{Resumen}

Nota preliminar de una investigación que tiene como objetivo proponer un programa de Educación Permanente en Salud a los técnicos responsables de los exámenes inmunohematológicos del Hemocentro Niterói, ubicado en el Hospital Universitario Antonio Pedro, a partir de una investigación con enfoque metodológico cualitativo de tipo descriptivo, con la naturaleza exploratório. La investigación obtuvo una opinión favorable del Comité de Ética en Investigación de la Universidad Federal Fluminense. La recolección de datos se realizará a través de cuestionarios semiestructurados. Los resultados serán analizados de acuerdo con la categorización y el análisis de contenido propuesto por Bardin. Como resultado, se pretende, a través de la Educación Permanente en Salud, proporcionar a los profesionales técnicos en hemoterapia nuevas formas de pensar y resolver los problemas que surgen en el trabajo diario, con el fin de aumentar la calidad del servicio prestado y la seguridad del paciente que está siendo tratado.

Palabras clave: Transfusión de Sangre; Servicio de Hemoterapia; Educación Continua.

1*Farmacêutica. Mestranda do Mestrado Profissional em Ensino na Saúde/MPES, Universidade Federal Fluminense, Niterói, RJ, Brasil. E-mail: milena_dias@id.uff.br ORCID: https://orcid.org/0000-0002-8505-2146

${ }^{2}$ Farmacêutico. Doutor. Professor do Programa de Mestrado Profissional em Ensino na Saúde/MPES, UFF, RJ, Brasil. E-mail: bcordeiro@id.uff.br ORCID: https://orcid.org/00000001-6387-511X 


\section{Introdução}

O presente estudo surgiu através do olhar diferenciado da pesquisadora sobre o enfrentamento dos diversos problemas da prática do trabalho apresentados a ela pelos técnicos de hemoterapia do Hemocentro Regional, localizado no Hospital Universitário Antônio Pedro (HUAP), em Niterói. Olhar esse conquistado pela realização do curso de Pós-graduação em Imunohematologia e pelo cargo de farmacêutica coordenadora da equipe técnica.

A pesquisadora verificou através do seu cargo de coordenação que; além da realização de treinamentos, implementação de protocolos e supervisão; era exigida a resolução, correção e prevenção dos problemas que pudessem surgir no cotidiano do setor. Por meio desta prática laboral, foi constatada a necessidade de mecanismos e metodologias que contassem com a participação da equipe para a resolução dos problemas, permitindo tanto o desenvolvimento da instituição, quanto daqueles que estão envolvidos.

O Hemocentro de Regional de Niterói é responsável por dar suporte às unidades de saúde de Niterói que realizam transfusões sanguíneas, o mesmo também é responsável pela captação de doadores, coleta de sangue, produção de hemocomponentes, testes prétransfusionais e pelas transfusões realizadas do HUAP.

Os benefícios da transfusão sanguínea são conhecidos desde o século XIX, porém com muitas reações adversas. ${ }^{1}$ Desde então, a medicina mundial transfusional não parou de evoluir, e no Brasil não foi diferente. Muitas normas e leis, ao longo desses anos, foram sendo criadas, com o objetivo de promover a segurança na transfusão., ${ }^{2,3}$

No Brasil, em 2017, foram realizados aproximadamente 2 milhões de transfusões de hemocomponentes, com cerca de 4 milhões de doações sanguíneas, concluindo-se o quanto é significativo esse procedimento para a saúde pública brasileira. ${ }^{4}$

Os testes imunohematológicos executados em um banco de sangue são extremamente importantes para a realização de uma transfusão sanguínea de sucesso, pois são eles que irão diagnosticar se uma bolsa de sangue será compatível ou não para um paciente. ${ }^{5}$

$\mathrm{Na}$ medicina transfusional, profissionais competentes e bem treinados tornam-se requisitos primordiais para a prevenção de possíveis complicações e reações transfusionais. Estudos demonstram que uma das causas que podem acarretar diminuição da segurança transfusional e prejuízos aos pacientes é a falta de conhecimento técnico e prático pelos profissionais de hemoterapia. ${ }^{6}$

Em 13 de fevereiro de 2004, o Ministério da Saúde (MS) instituiu a Portaria $\mathrm{n}^{\circ} 198$, que constituiu a Política Nacional de Educação Permanente em Saúde
(PNEPS), que possui intuito de aumentar a qualidade do serviço prestado à população através do financiamento de ações que capacitem os profissionais da saúde, com embasamento teórico-prático. Desse modo, utiliza-se a educação permanente em saúde (EPS) como estratégia para mudanças no trabalho em saúde, tornando o mesmo um ambiente de crítica reflexiva, construtivista, com compromisso e tecnicamente competente. ${ }^{7}$

As propostas educativas, relativas à EPS, são criadas e desenvolvidas a partir dos problemas evidenciados no processo de trabalho e na utilização de diferentes fundamentos educativos, principalmente, a aprendizagem significativa. Na PNEPS, a aprendizagem significativa é um dos conceitos educativos difundidos, onde existe uma maior valorização das experiências adquiridas do educando, fazendo com que o mesmo tenha um papel mais ativo no processo educativo. ${ }^{8}$

A EPS contribui para que a equipe reflita, pense e aja. Rompendo com a ideia do ensino transmissor por meio do ensino problematizador, baseado em ensinoaprendizagem, tornando a equipe mais comprometida com os atos de saúde. ${ }^{9}$

Neste intuito, o presente estudo trará como produto um plano de Educação Permanente em Saúde para os técnicos de hemoterapia com o propósito de problematizar e solucionar dúvidas ou intercorrências que venham surgir na rotina do setor, através de uma proposta aprendizagem-trabalho.

Objeto de estudo: a educação permanente da equipe de técnicos responsáveis pelos exames de imunohematologia do Hemocentro do Hospital Universitário Antônio Pedro.

Questões de pesquisa: Quais as dificuldades e problemas são enfrentados pela equipe na rotina do setor de hemoterapia? Qual a percepção da equipe sobre EPS? Quais estratégias educativas podem ser utilizadas para aplicar a EPS?

$\mathrm{O}$ estudo tem como objetivo geral propor um programa de Educação Permanente em Saúde aos técnicos do setor de hemoterapia do Hospital Universitário Antônio Pedro. E como objetivos específicos: 1- Verificar a percepção dos técnicos de hemoterapia sobre Educação Permanente em Saúde; 2- Identificar a melhor estratégia educativa para a aplicação de educação permanente em saúde; 3-Analisar as principais dificuldades encontradas nas práticas do cotidiano do setor de hemoterapia; 4- Apresentar um plano de Educação Permanente em Saúde como produto para os técnicos de hemoterapia.

\section{Método}

Trata-se de uma nota prévia de um estudo com abordagem metodológica qualitativa do tipo descritiva, com natureza exploratória. Como instrumento de coleta 
de dados será realizada uma entrevista individual, que será guiada por um roteiro semiestruturado e para a caracterização de dados será aplicado um questionário, com gravação e transcrição na íntegra.

A abordagem principal será qualitativa que serve para entender e aprofundar ideias sobre os sujeitos analisados, tratando a situação como única, baseandose nas suas experiências, suas vivências. Esse tipo de pesquisa é baseado na especificidade do fenômeno estudado. O pesquisador deve ser o mais imparcial possível. ${ }^{10}$

O cenário da pesquisa seráo Hemocentro Regional de Niterói, localizado no Hospital Universitário Antônio Pedro. Atualmente, o HUAP é a maior e mais complexa unidade de saúde da Grande Niterói, sendo uma unidade de saúde de alta complexidade. A mesma abrange uma área em torno de dois milhões de habitantes. Sua missão é gerar, transformar e difundir o conhecimento, prestando serviços de saúde com excelência, de forma digna, crítica e hierarquizada.

Serão incluídos no estudo os técnicos responsáveis pelos exames imunohematológicos, lotados no setor, estatutários e que aceitarem participar do projeto. Como critério de exclusão do estudo os técnicos que estiverem de licença médica, licença prêmio ou usufruindo de férias.

A pesquisa obteve parecer favorável pelo comitê de Ética em Pesquisa da Universidade Federal Fluminense (CAAE: 17559319.5.0000.5243). A participação do estudo será voluntária, os participantes serão convidados a participar da entrevista e assinarão o termo de Consentimento Livre Esclarecido conforme Resoluções $\mathrm{n}^{\circ}$ 466/12, 510?2016 e 580/2018 do Conselho Nacional de Saúde (CNS).

Os resultados das questões abertas sobre o que os técnicos entendem de educação permanente em saúde e quais são as principais dificuldades encontradas na prática serão analisados conforme categorização e análise de conteúdo proposta por Bardin. A escolha para o tema inicial abordado e a estratégia educativa utilizada na aplicação da educação permanente será feita por análise estatística.

Sendo esse um mestrado profissional em ensino e tendo sua linha de pesquisa em Educação Permanente em Saúde, a elaboração de um produto faz-se necessária visando à mudança do cenário estudado. Então será criado um Plano de Educação Permanente em Saúde para os técnicos de hemoterapia.

\section{Resultados esperados}

Como contribuição pretende-se proporcionar aos profissionais técnicos em hemoterapia novas maneiras de pensar e solucionar os problemas surgidos no cotidiano do trabalho. Através da discussão, reflexão, problematização e valorização das experiências dos profissionais envolvidos na pesquisa espera-se que ocorra um aprendizado significativo, com a finalidade de aumentar a qualidade do serviço prestado e a segurança do paciente atendido.

\section{Referências}

1. Brasil. Agência Nacional de Vigilância Sanitária. Hemovigilância: manual técnico para investigação das reações transfusionais imediatas e tardias não infecciosas / Agência Nacional de Vigilância Sanitária. - Brasília: Anvisa, 2007.

2. Junqueira PC, Rosenblit J, Hamerschlak N. História da Hemoterapia no Brasil. Rev. bras. hematol. hemoter. 2005; 27(3): 201-207.

3. Júnior JBS, Rattner D. A Vigilância Sanitária no controle de riscos potenciais em serviços de hemoterapia no Brasil. Saúde Debate | rio de Janeiro. 2016; 109 (40): 136-153.

4. Brasil. Agência Nacional de Vigilância Sanitária. $6^{\circ}$ Boletim de produção hemoterápica hemoprod 2017. - Brasília: Anvisa, 2018

5. Flausino GF, et al $\mathrm{O}$ ciclo de produção do sangue e a transfusão: o que o médico deve saber. Rev. Med. Minas Gerais. 2015; 25(2): 269-279.

6. Silva KFN, Soares S, Iwamoto HH. A prática transfusional e a formação dos profissionais de saúde. Rev Bras Hematol Hemoter. 2009; 31(6): 421-426.

7. Puggina CC. et al. Educação permanente em saúde: instrumento de transformação do trabalho de enfermeiros. Rev Esp Saúde. 2015; 16(4): 8797.

8. Silva LAA, et al. Educação permanente em saúde na atenção básica: percepção dos gestores municipais de saúde. Rev. Gaúcha Enferm. 2017; 38(1): e58779.

9. Figueiredo EBL, Gouvêa MV, Silva ALA. Educação Permanente em Saúde e Manoel de Barros: uma Aproximação Desformadora. Revista Brasileira de Educação Médica. 2016; 40(3): 324-331.

10. Minayo MCS. Amostragem e saturação em pesquisa qualitativa: consensos e controvérsias. Revista Pesquisa Qualitativa. 2017; 5(7): 01-12 